\title{
ROLE OF THERAPEUTIC PLASMA EXCHANGE IN THE COMPLEX TREATMENT OF SEVERE KIDNEY DISEASES
}

\author{
Alexander Stoyanov, Krasimira Nenova, Kiril Nenov \\ Department of Internal Medicine, Medical University of Varna
}

\begin{abstract}
PURPOSE: The strongest effect of therapeutic plasma exchange (TPE) is achieved in diseases with immune and autoimmune genesis, hyperviscous conditions and intoxications of various origins. The aims of the study were to assess the long-term effect of TPE in the complex therapy of severe renal diseases and to highlight therapeutic strategies necessary to achieve good clinical results.

MATERIAL AND METHODS: During the period 1980-2010, TPE was carried out in the form of a complex treatment of 87 patients with immune nephropathies and 44 patients with malignant myeloma. The primary diseases were the following: chronic glomerulonephritis - 42, lupus nephropathy - 31, Henoch-Schönlein nephritis - 8, Wegener granulomatosis - four and Goodpasture syndrome - two patients. The diagnosis was confirmed after a puncture kidney biopsy in $72(82,8 \%)$ patients with immune nephropathies. TPE was performed using centrifugal or filtration methods.
\end{abstract}

RESULTS: The patients with immune nephropathies underwent between 5 and 7 consecutive plasma exchanges daily or every two days. A three consecutive days of corticosteroid pulse therapy was initiated after last procedure followed by conventional immunosuppressive therapy. After serial TPE a significant decrease in anti-GMB antibodies and circulating immune complexes was detected. Continuous clinical and paraclinical remission was achieved in $62,7 \%$ of the patients who received the combined treatment.

CONCLUSION: TPE has a beneficial effect after being included in the complex treatment of patients with severe immune nephropathies. The immunomodulation contributes to the successful management of severe ongoing autoimmune processes. In patients with hyperviscosity, 'supporting' TPE every 2 or 3 months is required to lower the increased viscosity, to prevent thromboembolic complications, and to slow-down nephropathy progression.

Key words: therapeutic plasma exchange, plasmapheresis, immune nephropathies, malignant myeloma, immunosuppression

Address for correspondence:

Alexander Stoyanov, MD, PhD

Department of Internal Medicine,

Medical University of Varna

55 Marin Drinov Str.,

9002 Varna, Bulgaria

E-mail:pv3ss@abv.bg

Received: December 4, 2012

Accepted: January 15, 2013

\section{INTRODUCTION}

Therapeutic plasma exchange (TPE) is a process involving the extracorporeal separation of plasma from the cellular components of blood, discarding the plasma and exchanging it with replacement physiologic fluids such as albumin or fresh frozen plasma to maintain oncotic pressure and blood volume, and then returning this and the original cellular components of blood back to the patient's circulatory system $(4,5,7,19)$.

TPE is a extracorporeal therapy commonly used in the treatment of a variety of neurological, renal, 
hematological, and other systemic diseases caused by circulating 'toxic agents' that can't be cleared by other means. Most authors report effectiveness in about $50 \%$ of cases. The strongest effect is achieved in diseases with immune and autoimmune genesis, hyperviscous conditions and intoxications of various origins $(1,10)$.

As plasma is used as the replacement ('exchange') fluid for a limited number of pathological conditions, 'plasmapheresis' is a more precise term than 'therapeutic plasma exchange', the latter of which implies not only plasma removal, but also replacement with plasma. Occasionally, the appropriate replacement fluid is colloid and/ or crystalloid instead of plasma. Because the use of 'TPE' is more common, it is used synonymously with plasmapheresis (3).

Many primary renal diseases are associated with either antibody deposition within the glomerulus or an antibody associated autoimmunity, as may be seen with certain vasculitidies. Other immunoglobulins may be nephrotoxic or glomerulopathic (16). Here belongs the case with myeloma-related light chains or cryoglobulins.

The goal of plasma exchange is to mechanically extract pathogenic autoantibodies, immune complexes, cryoglobulins, myeloma light chains, endotoxin, cholesterol-containing lipoproteins and other unknown mediators. The basic premise of TPE is that removal of certain pathologic substances from the plasma will reduce further damage and may permit reversal of the pathologic process. Other potential benefits include unloading of the reticuloendothelial system (thereby enhancing endogenous removal of the circulating toxins) (18), stimulation of lymphocyte clones to enhance cytotoxic therapy (17), and the possibility of reinfusing large volumes of plasma without the risk of intravascular volume overload.

In order to achieve the necessary immunomodulation it is required to carry out multiple consecutive TPE procedures. The plasma exchange has to be followed by immunosuppression in order to avoid the undesirable rebound effect which could compromise the achieved decrease in antibodies. Patients with counterindications for continuous immunosuppression report increased TPE effectiveness.

Dysproteinemias consist of a broad range of severe pathological states with the common thread of excessive production of an abnormal protein or paraprotein. Various clinical syndromes may arise, either from the underlying disease process, the excess paraprotein, or both. Clinical presentation depends upon the organ systems affected by the abnormal protein. Diseases included under the classification of dysproteinemias include cryoprotein-related diseases, Waldenström's macroglobulinemia, hyperviscosity syndrome, monoclonal gammopathy, multiple myeloma (15), light chain disease, and amyloidosis. Plasmapheresis, often in conjunction with other therapies, is widely used to treat the dysproteinemias and their resulting clinical syndromes. Automated plasmapheresis that separates plasma from the cellular blood elements by centrifugation is used most commonly in the United States. Membrane separation and immunoadsorption techniques are more commonly used in Europe and Japan $(2,8)$. Membrane separation and immunoadsorption allow the offending proteins to be removed more selectively from the patient's plasma prior to the plasma being returned to the patient.

The purposes of the present study were to evaluate TPE long-term effect in the complex therapy of severe renal diseases and to highlight therapeutic strategies necessary to achieve good clinical results.

\section{MATERIAL AND METHODS}

During the period 1980-2010 in the Clinic of Nephrology and Hemodialysis, St. Marina University Hospital of Varna $(11,12)$, TPE was carried out in the form of a complex treatment in 87 patents, 38 males and 49 females, with immune nephropathies (Table 1). Two methods of TPE were applied: centrifugal (with a Hemonetix M30 apparatus) and plasma filtration (with plasma filters PLASMAFLUX P-1 and P-2). During the procedures, $1314 \pm 285 \mathrm{~mL}$ of plasma were extracted on average. Fluid replacement was achieved with plasma and colloid or crystalloid fluids in relatively equal proportions. This method was also applied to 44 patients, 31 males and 13 females, with malignant myeloma with the intention 
Table 1. Patient's distribution according to their primary disease

\begin{tabular}{c|l|c|}
\hline No & \multicolumn{1}{|c|}{ Diagnosis } & $\mathrm{n}$ \\
\hline 1. & Chronic glomerulonephritis & 42 \\
2. & Lupus nephropathy & 31 \\
3. & Henoch-Schönlein nephritis & 8 \\
4. & Wegener granulomatosis & 4 \\
5. & Goodpasture syndrome & 2 \\
& Total & 87 \\
\hline \hline
\end{tabular}

to lower the concentration of pathological proteins, to diminish the pathologically increased plasma viscosity (in order to enhance of microcirculation and to prevent vascular thrombosis) and to delay the development of myeloma nephropathy. More plasma per procedure was extracted in the treatment of these patients (an average of $2014 \pm 367 \mathrm{~mL}$ ). The fluid replacement of plasma was achieved mainly by using crystalloid fluids. The diagnosis was confirmed after a puncture kidney biopsy in $72(82,8 \%)$ patients with immune nephropathies. Kidney biopsies were not performed on patients with advanced renal failure.

\section{RESULTS}

Table 2 shows the patients according to their renal function.

Table 2. Patient's distribution according to their renal function

\begin{tabular}{c|l|c|}
\hline No & Renal function & $\mathrm{n}$ \\
\hline 1. & Normal renal function & 61 \\
2. & Early stages of renal failure (1-2 stage) & 12 \\
3. & Advanced renal failure & 14 \\
\hline
\end{tabular}

In the patients with immune nephropathies 5 to 7 consecutive plasma exchanges were carried out daily or every two days. A three consecutive days of corticosteroid pulse therapy was initiated after last procedure and was followed by conventional immunosuppressive therapy $(18,19)$.

In the patients with Wegner's granuloma, 7 to 9 procedures were necessary in order to achieve the desirable effect $(1,2)$. Most TPE (6) were carried out on two patients with Goodpasture syndrome (9), with 10 and 12 procedures, respectively.

After finishing the plasma exchanges a significant decrease in anti-GMB antibodies was detected along with a decrease in circulating immune complexes (Fig. 1).

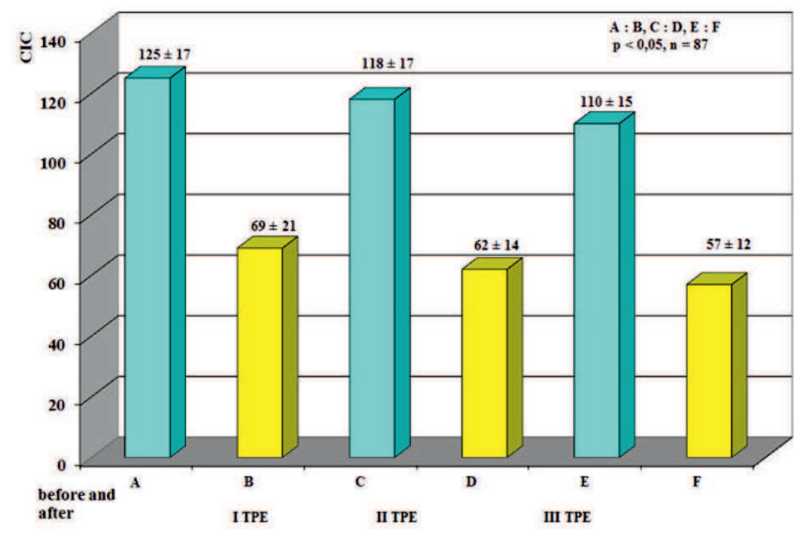

Fig. 1. CIC values before and after serial TPE in patients with immune nephropathies

Improved phagocytic activity of polymorphonuclear leukocytes after serial TPE was shown on Fig. 2. Continuous clinical and paraclinical remission was achieved in $62,7 \%$ of the patients on combined treatment in a timely manner. Additionally, delayed renal failure progression was reported with these patients compared to the control group (patients not undergoing TPE) $(13,16)$.

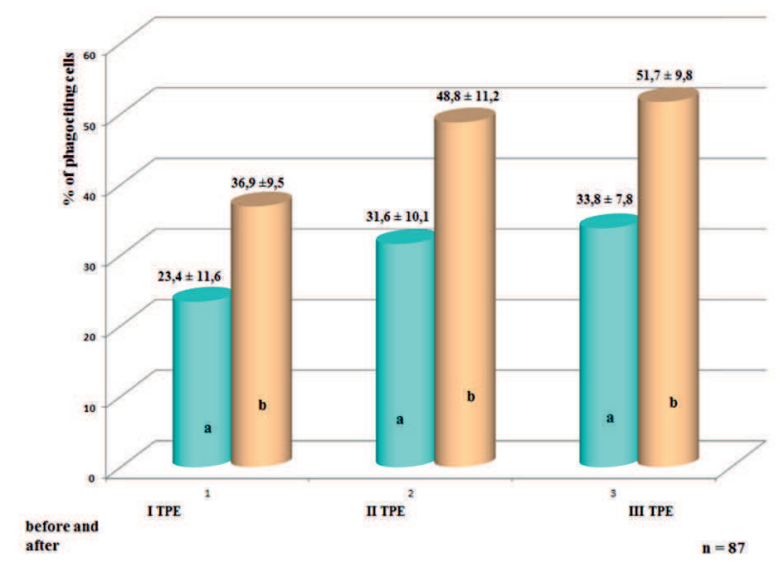

Fig. 2. Phagocite activity of polymorphonuclear cells before and after serial TPE in patients with immune nephropathies 
After completing TPE, a significant decrease in pathologically high plasma viscosity was detected in patients with myeloma nephropathy (Fig. 3).

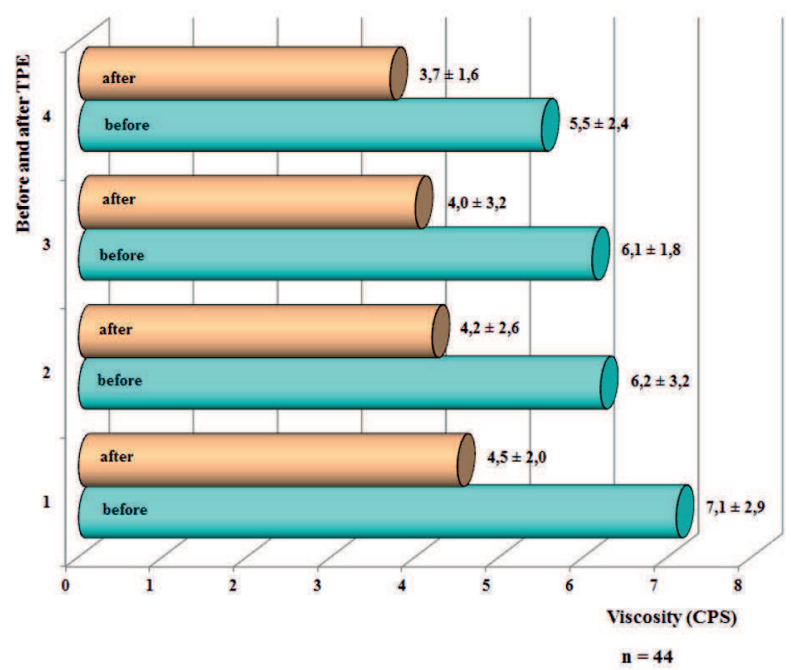

Fig. 3. Plasma viscosity before and after serial TPE in patients with multiple myeloma and hyperviscosity

Additionally, the biological toxicity test indicated TPE pronounced detoxicating abilities (15) (Fig. 4).

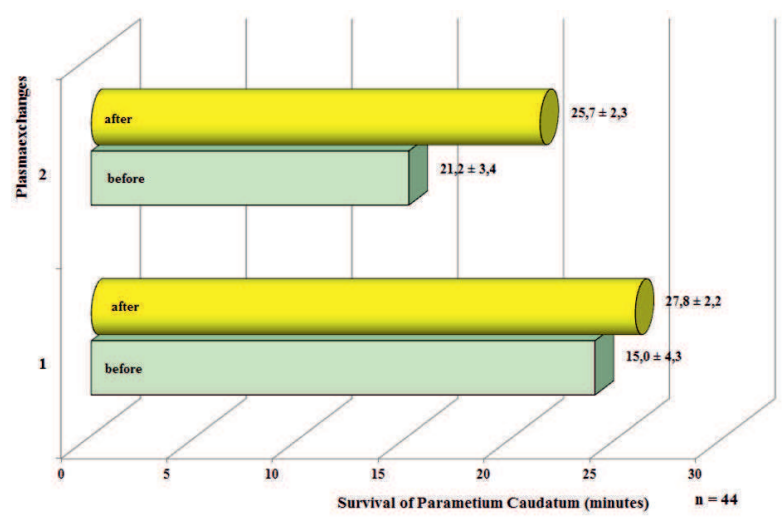

Fig. 4. Plasma toxicity before and after serial TPE in patients with multiple myeloma

\section{DISCUSSION}

This evidence corroborates the theory that early-stage renal failure, before the onset of permanent irreversible changes in kidneys, can be managed with combined treatment. Patients at stage 3 and stage 4 renal failure do not demonstrate any desirable effects. Most of them shortly afterwards began hemodialysis treatment.

\section{CONCLUSION}

Based on our findings and literature data available the conclusion can be drawn that the TPE has a beneficial effect after being included in the complex treatment of patients with severe immune nephropathies. The achieved immunomodulation contributes to the successful management of severe ongoing autoimmune processes. In patients with hyperviscosity, it is necessary to conduct 'supporting' TPE (14) every 2 or 3 months in order to reduce the increased viscosity, to prevent thromboembolic complications, and to slow-down nephropathy progression.

\section{REFERENCES}

1. Kaplan, A. A. Therapeutic plasma exchange: core curriculum.- Am. J. Kidney Dis., 52, 2008, No 6, 1180-1186.

2. Drew, M. J. Plasmapheresis in the dysproteinemias.- Ther. Apher., 6, 2002, No 1, 45-55.

3. Fridey, J. L, A. A. Kaplan. Indications for therapeutic plasma exchange. - Up to Date, 2012.

4. Ibrahim, R. B., R. A. Balogun. Therapeutic apheresis.- Semin. Dial., 25, 2012, 176-189. 5.

5. Ibrahim, R. B., R. A. Balogun. Medications and therapeutic apheresis procedures: Are we doing our best?- J. Clin. Apher., 28, 2013, No 1, 73-77.

6. Jiao, L. P., J. F. Fan, Q. Sun, Y. Shen. Plasma exchange in Goodpasture syndrome associated with Turner's syndrome: A case report.- Afr. Health Sci., 4, 2012, 572-575.

7. Kaplan, A. A. Therapeutic apheresis for renal disorders.- Therap. Apher., 3, 1999, No 1, 25-30.

8. Kes, P. Therapeutic plasma exchange.- Acta Med. Croat., 53, 1999, No 3, 129-139.

9. Lockwood, C., J. Jones, R. Lowenthal, et al. Recovery from Goodpasture's syndrome after immunosuppressive treatment and plasmapheresis.- Brit. Med. J., 2, 1975, 252-254.

10. Madore, F., J. M. Lazarus, H. R. Brady. Therapeutc plasma exchange in renal diseases.- J. M. Soc. Nephrol., 7, 1996, 367-386.

11. Nenov, D., I. Stanchev, P. Chankova. First results from the treatment of glomeruloonephriotis with 
plasmaphesresis.- Vutreshni bolesti, 20, 1981, No 2, 97-105 (in Bulgarian).

12. Nenov, D., I. Stanchev, M. Gardevski, D. Paskalev, K. Nenov. Treatment of Wegener granulomatosis with plasmapheresis.- Vutreshni bolesti, 22, 1983, No 6, 58-61 (in Bulgarian).

13. Nenov, K., D. Nenov. Treatment of renal diseases with plasmapheresis.- Medicinska Istrazivanja, 33, 1999, 68-70.

14. Nenov D., K. Nenov. Therapeutic Apheresis in exogenous poisoning and in myeloma.- Nephrol. Dial. Transplant., 16, 2001, Suppl. 6, 101-102.

15. Nenov, K., D. Nenov, V. Nenov. Our experience using different methods to verify the detoxification effect of plasma exchange (PE).- Int. J. Art. Org., 20, 1997, No 2, 125-126.

16. Niwa, T., M. Ise. Indoxyl sulfate, a circulating uremic toxin, stimulates the progression of glomerular sclerosis.- J. Lab. Clin. Med., 124, 1994, No 1, 96-104.
17. Smith, J. W., R. Weinstein, K. L. Hillyer. Therapeutic apheresis: a summary of current indication categories endorsed by the AABB and the American Society for Apheresis.- Transfusion, 43, 2003, No 6, 820-824.

18. Szczepiorkowski, Z.M., J. L. Winters, N. Bandarenko, H. C. Kim, M. L. Linenberger, M. B. Marques, R. Sarode, J. Schwartz, R. Weinstein, B. H. Shaz. Guidelines on the use of therapeutic apheresis in clinical practice - evidence-based approach from the Apheresis Applications Committee of the American Society for Apheresis.J. Clin. Apher., 25, 2010, No 3, 83-88.

19. Winters, J. L. Apheresis medicine: state of the art in 2010: American Society for Apheresis fifth special edition of the Journal of Clinical Apheresis.- $J$. Clin. Apher., 26, 2011, No 5, 239-242. 